CRYSTALLOGRAPHIC COMMUNICATIONS

ISSN 2056-9890

Received 12 July 2017

Accepted 18 July 2017

Edited by $\mathrm{H}$. Stoeckli-Evans, University of Neuchâtel, Switzerland

Keywords: crystal structure; ordering phase transition; reversible phase transition; nonmerohedral twinning; $\mathrm{C}-\mathrm{H} \cdots \pi$ interactions.

CCDC references: 1563032; 1563031

Supporting information: this article has supporting information at journals.iucr.org/e

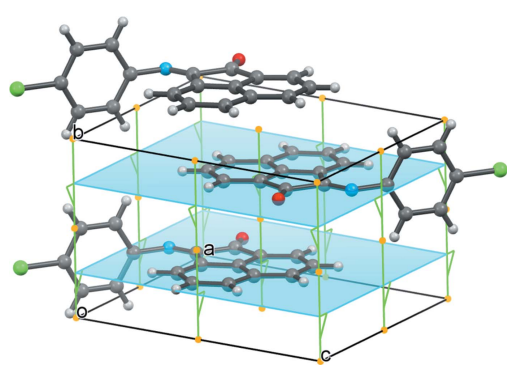

OPEN $\odot$ ACCESS

\section{A temperature-dependent phase transformation of (E)-2-[(4-chlorophenyl)imino]acenaphthylen-1-one}

\author{
Lipiao Bao ${ }^{a}$ and Marilyn M. Olmstead ${ }^{b *}$
}

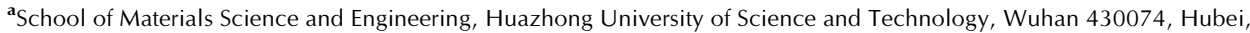
China, and ${ }^{\mathbf{b}}$ Department of Chemistry, University of California, Davis, CA 95616, USA. *Correspondence e-mail: mmolmstead@ucdavis.edu

The crystal structure determination based on $90 \mathrm{~K}$ data of the title imine ligand, $\mathrm{C}_{18} \mathrm{H}_{10} \mathrm{ClNO}$, revealed non-merohedral twinning with three twin domains. In our experience, this is an indication of an ordering phase transition. Consequently, the structure was redetermined with higher temperature data, and a reversible phase transition was discovered. The higher temperature phase is indeed an ordered structure. At the higher temperature, the 4-chlorophenyl group has rotated by $c a 7^{\circ}$ into a crystallographic mirror plane. Warming the crystal from $90 \mathrm{~K}$ to $250 \mathrm{~K}$ changes the space group from triclinic $P \overline{1}$, to monoclinic $P 2_{1} / m$. Diverse non-classical interactions are present in the crystal packing, and these are described for the phase change reported in this work. The crystal structure of the title imine ligand, measured at $100 \mathrm{~K}$, has been reported on previously [Kovach et al. (2011). J. Mol. Struct. 992, 33-38].

\section{Chemical context}

Transition metal complexes that can photochemically release carbon monoxide upon exposure to visible light have been reported recently (Chakraborty et al., 2014; Stenger-Smith et al., 2017). Facile release of carbon monoxide has been observed in manganese carbonyls containing acenaphthalene derivatives (Carrington et al., 2015) including the ligand MIAN \{2-[(4-chlorophenyl)imino]acenapthylen-1-one\}, the subject of this study, shown in the Scheme. Our crystal structure determination of MIAN at $90 \mathrm{~K}$ agrees with the structure reported by Kovach et al. (2011) at $100 \mathrm{~K}$. In particular, the structure occurs in the triclinic space group $P \overline{1}$ and it is found to be a twin. In the NMR study of MIAN by Kovach et al., major and minor species were detected in $\mathrm{CDCl}_{3}$ at room temperature and a single species at $388 \mathrm{~K}$ in DMSO- $d_{6}$. They suggested that an $E$ to $Z$ equilibration with the $E$ form dominant takes place at the elevated temperature. The occurrence of a low-symmetry space group and twinning are indicative of a solid-solid phase change, and we were curious about the structure at higher temperatures. While a change of conformation from $E$ to $Z$ would be a very large solid-state change, an alternative structural change would be possible. At $250 \mathrm{~K}$, a small solid-state change was indicated and the new space group is $P 2_{1} / m$ ( $\alpha$ phase). The only difference, aside from small differences in unit-cell dimensions, is a rotation of the iminoacenapthylen-1-one group into a crystallographic mirror plane. In each phase, the molecule remains in the $E$ conformation. 


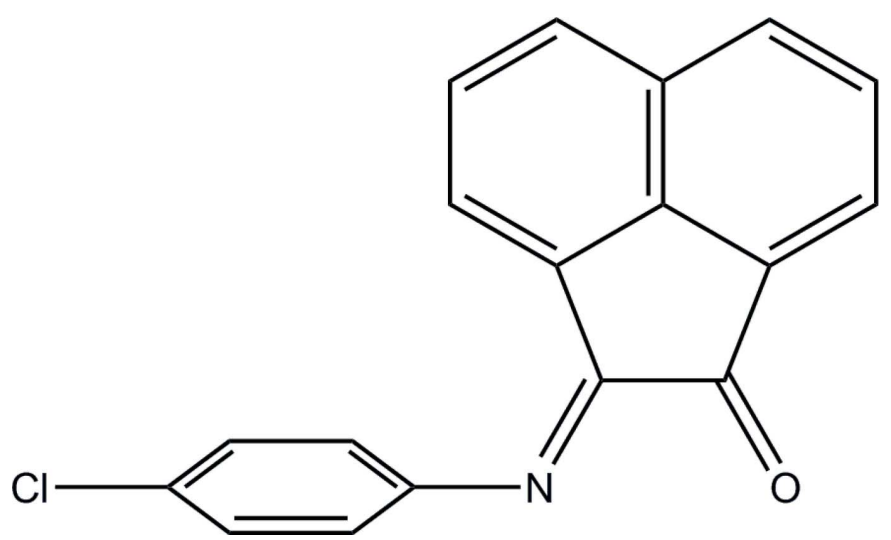

\section{Structural commentary}

The crystal structure was initially determined at $90 \mathrm{~K}$. Three twin domains were found, with relative contributions of 0.441 (2), 0.058 (3), 0.060 (3). Redetermination of the structure at higher temperatures validated our suspicion that the structure was temperature-sensitive. In order to more easily compare the low-temperature and room-temperature crystal structures, a non-standard setting for the triclinic form was selected. In this setting the shortest axis is the $b$ axis. The $b$ axis is then the unique axis in the monoclinic setting of $P 2_{1} / \mathrm{m}$. Since minor changes in unit-cell dimensions occur, the exact temperature of the phase change was difficult to determine, but examination of the diffraction images revealed obvious twinning between 90 and $208 \mathrm{~K}$, coalescence of diffraction spots occurring at $230 \mathrm{~K}$, and by $250 \mathrm{~K}$ it was clear that the twinning had vanished and the space-group symmetry had changed. Solution of the two structures showed that the structural effect of the temperature change goes from triclinic, $P \overline{1}$ with $Z=2\left(Z^{\prime}=1\right)$ to monoclinic, $P 2_{1} / m$ with $Z=2\left(Z^{\prime}=\right.$ $0.5)$. The most obvious structural change involves rotation and

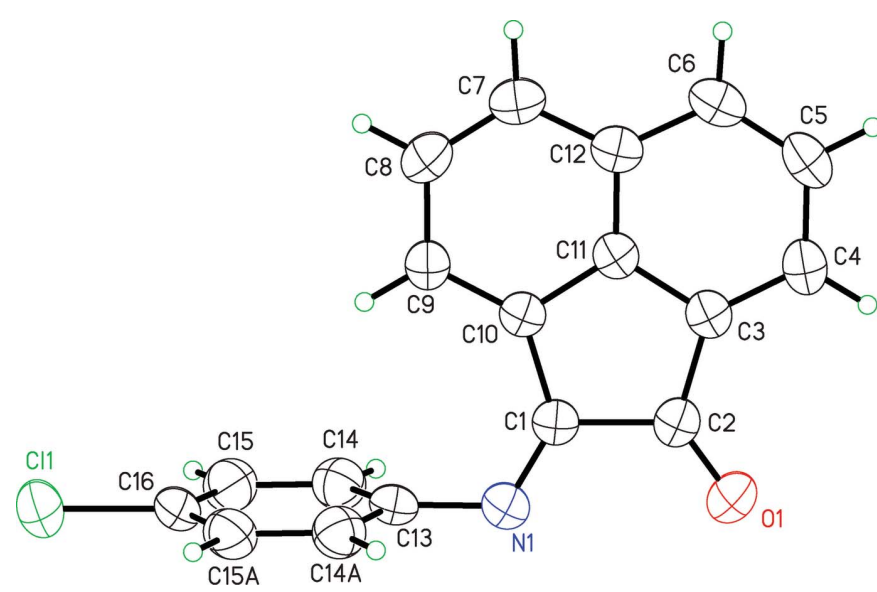

Figure 1

Molecular structure of the title compound at $250 \mathrm{~K}$ ( $\alpha$-phase), showing $50 \%$ thermal displacement parameters and the atom-numbering scheme. Atoms $\mathrm{C} 14$ and $\mathrm{C} 15$ are related related to atoms $\mathrm{C} 14 \mathrm{~A}$ and $\mathrm{C} 15 \mathrm{~A}$, respectively, by mirror symmetry.
Table 1

Hydrogen-bond geometry $\left(\AA,^{\circ}\right)$ for the $\alpha$-phase.

$\mathrm{Cg}$ is the centroid of the 4-chlorophenyl ring (C13-C16/C14A/C15A).

\begin{tabular}{lllll}
\hline$D-\mathrm{H} \cdots A$ & $D-\mathrm{H}$ & $\mathrm{H} \cdots A$ & $D \cdots A$ & $D-\mathrm{H} \cdots A$ \\
\hline $\mathrm{C} 6-\mathrm{H} 6 \cdots C g^{\mathrm{i}}$ & 0.94 & 2.86 & $3.803(2)$ & 177 \\
$\mathrm{C} 9-\mathrm{H} 9 \cdots C g$ & 0.94 & 2.88 & $3.668(11)$ & 128 \\
\hline
\end{tabular}

Symmetry code: (i) $x+1, y, z+1$.

Table 2

Hydrogen-bond geometry $\left(\AA{ }^{\circ}\right)$ for the $\beta$-phase.

$\mathrm{Cg}$ is the centroid of the 4-chlorophenyl ring (C13-C18).

\begin{tabular}{lllll}
\hline$D-\mathrm{H} \cdots A$ & $D-\mathrm{H}$ & $\mathrm{H} \cdots A$ & $D \cdots A$ & $D-\mathrm{H} \cdots A$ \\
\hline $\mathrm{C} 7-\mathrm{H} 7 \cdots \mathrm{Cl}{ }^{\mathrm{i}}$ & 0.95 & 2.80 & $3.748(2)$ & 179 \\
$\mathrm{C} 6-\mathrm{H} 6 \cdots C g^{\mathrm{ii}}$ & 0.95 & 2.75 & $3.698(4)$ & 177 \\
$\mathrm{C} 9-\mathrm{H} 9 \cdots C g$ & 0.95 & 2.87 & $3.644(4)$ & 142 \\
\hline
\end{tabular}

Symmetry codes: (i) $x, y, z+1$; (ii) $x+1, y, z+1$.

a change in the dihedral angle between the two molecular planes that brings the acenapthyl group into the crystallographic mirror plane. At $250 \mathrm{~K}$ the dihedral angle is $90^{\circ}$ while at $90 \mathrm{~K}$ it is $83.16(4)^{\circ}$. The unit-cell volume is $2.5 \%$ larger at the higher temperature. As would be expected, thermal motion is greater at high temperature, with $U_{\text {eq }}$ averaging $0.047 \AA^{2}$ vs $0.017 \AA^{2}$ at low temperature. Thermal motion in the 4-chlorophenyl ring is slightly greater than the acenapthyl group at both temperatures, $13.5 \%$ greater in the $\alpha$-phase (90 K) and $10.0 \%$ in the $\beta$-phase (250 K). Figs. 1 and 2 , depict the high ( $\alpha$-phase) and low ( $\beta$-phase) temperature structures, respectively. The similarity in the packing is evident from Figs. 3 and 4.

\section{Supramolecular features}

The two rings are perpendicular within each polymorph, likely due to a steric effect between $\mathrm{H} 9$, bonded to $\mathrm{C} 9$, and one of the ortho hydrogen atoms on the 4-chlorophenyl ring (with centroid $C g$ ). As a result of the perpendicular arrangement of

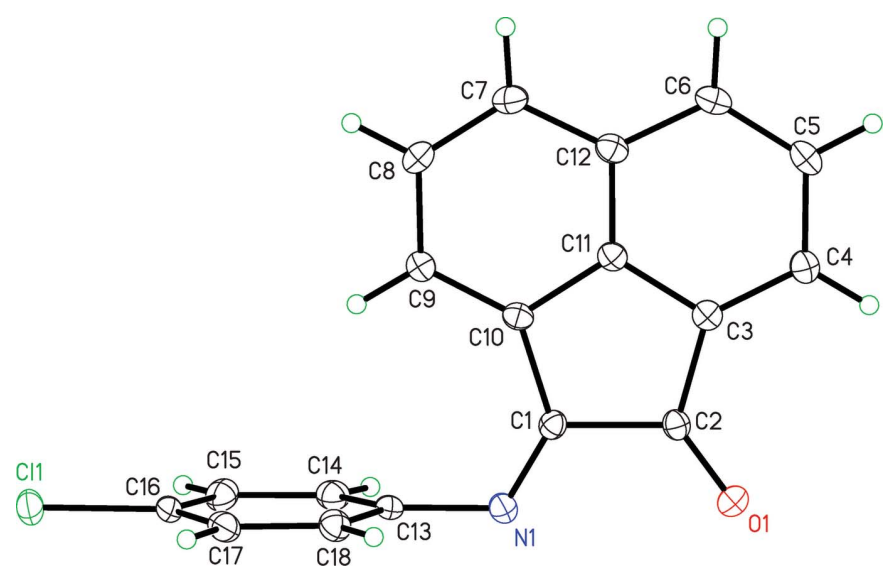

Figure 2

Molecular structure of the title compound at $90 \mathrm{~K}$ ( $\beta$-phase), showing $50 \%$ thermal displacement parameters and the atom-numbering scheme. 


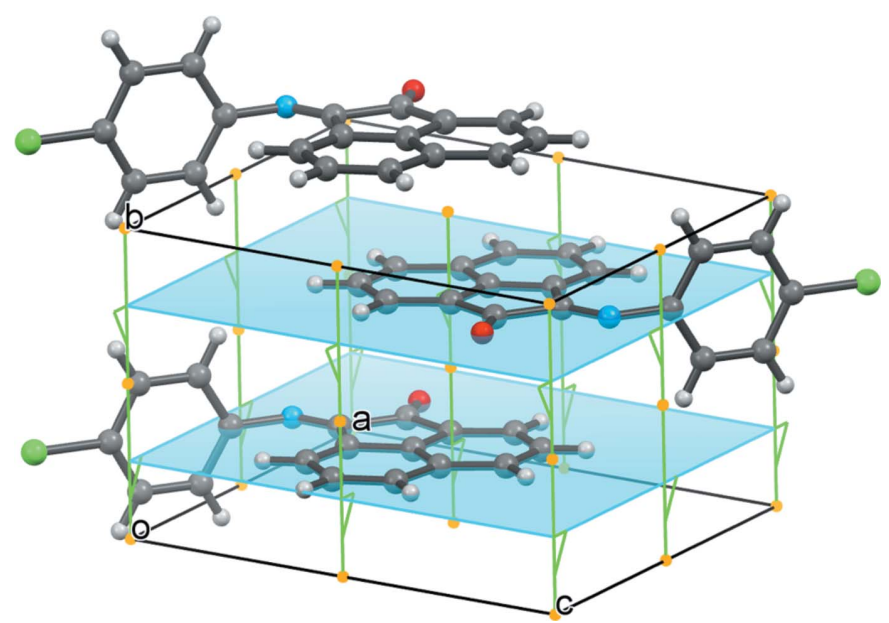

Figure 3

A view of the packing of the room temperature structure ( $\alpha$-phase). The crystallographic mirror planes are shown in blue. Orange dots indicate the crystallographic centers of inversion.

the two ring systems, there is an intramolecular $\mathrm{H} 9 \cdots \mathrm{Cg}$ distance of $2.90 \AA$ in the $250 \mathrm{~K}$ structure and $2.85 \AA$ in the $90 \mathrm{~K}$ structure (Tables 1 and 2). Neither structure has solventaccessible voids. We looked for intra- and intermolecular interactions that might be influential in the structural change. The only significant non-classical hydrogen bond of the $\mathrm{C}-$ $\mathrm{H} \cdot \cdots A$ type present is found in the crystal structure of the lowtemperature structure ( $\beta$-phase), with a $\mathrm{C}-\mathrm{H} \cdots \mathrm{Cl}^{\mathrm{i}}$ hydrogen bond linking neighbouring molecules to form chains along the

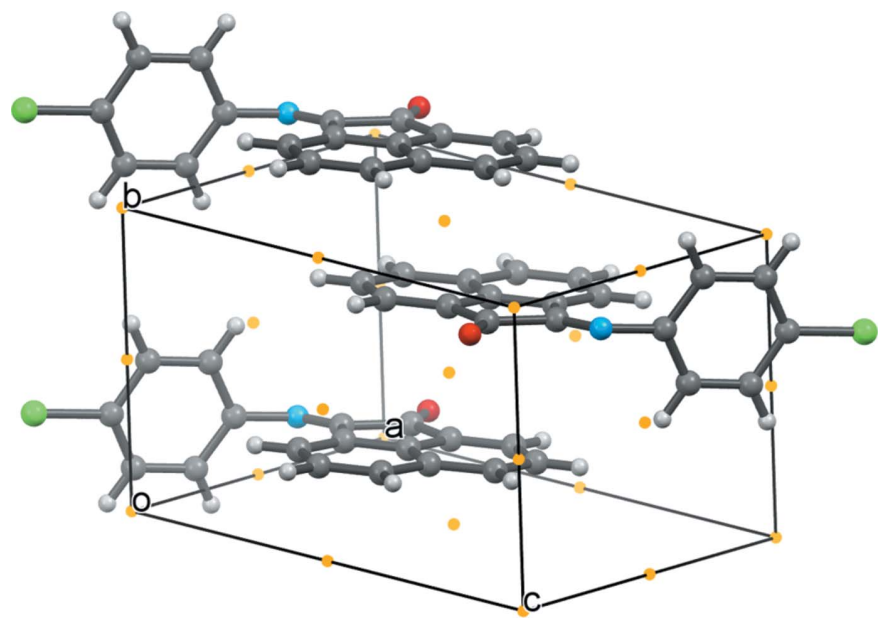

Figure 4

A view of the packing of the low temperature structure ( $\beta$-phase). Orange dots indicate the crystallographic centers of inversion.

$c$-axis direction (Table 2). There is, however, $\pi-\pi$ stacking between the acenapthyl groups in each case: the interplanar distance is $3.438 \AA$ at $250 \mathrm{~K}$ and $3.409 \AA$ at $90 \mathrm{~K}$. In both phases there is a $\mathrm{C}-\mathrm{H} \cdots \pi$ interaction on both sides of the phenyl ring, one intramolecular and one intermolecular (Tables 1 and 2, and Fig. 5). Temperature-driven phase changes such as this one that occur without major structural reorganization or ordering transitions have been reported in many cases: see, for example, Takahashi \& Ito (2010) and Takanabe et al. (2017) and references therein.

Table 3

Experimental details.

$\alpha$-phase

\section{Crystal data}

Chemical formula

$M_{\mathrm{r}}$

Crystal system, space group

Temperature $(\mathrm{K})$

$a, b, c(\AA)$

$\alpha, \beta, \gamma\left({ }^{\circ}\right)$

$V\left(\AA^{3}\right)$

$Z$

Radiation type

$\mu\left(\mathrm{mm}^{-1}\right)$

Crystal size (mm)

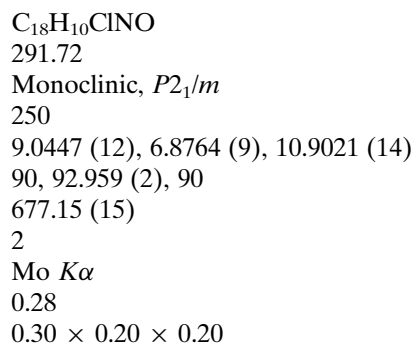

Bruker APEXII

Multi-scan (SADABS; Bruker, 2014)

$0.684,0.745$

$5458,1496,1227$

0.022

0.625

$0.039,0.097,1.04$

1496

121

$\mathrm{H}$-atom parameters constrained

$0.26,-0.38$ $\beta$-phase

$\mathrm{C}_{18} \mathrm{H}_{10} \mathrm{ClNO}$

291.72

Triclinic, $P \overline{1}$

90

9.0764 (10), 6.8187 (8), 10.7450 (12)

90.880 (2), 92.780 (2), 96.259 (2)

$660.12(13)$

2

Mo $K \alpha$

0.29

$0.30 \times 0.20 \times 0.20$

Bruker APEXII

Multi-scan (TWINABS; Bruker, 2014) $0.629,0.746$

$34083,2949,2726$

0.023

0.652

$0.031,0.092,1.08$

2949

193

$\mathrm{H}$-atom parameters constrained $0.33,-0.24$

$\mathrm{H}$-atom treatment
$\Delta \rho_{\max }, \Delta \rho_{\min }\left(\mathrm{e} \AA^{-3}\right)$

Computer programs: APEX2 and SAINT (Bruker, 2014), SHELXT (Sheldrick, 2015a), SHELXL2016/6 (Sheldrick, 2015b), SHELXTL (Sheldrick, 2008) and Mercury (Macrae et al., 2008). 


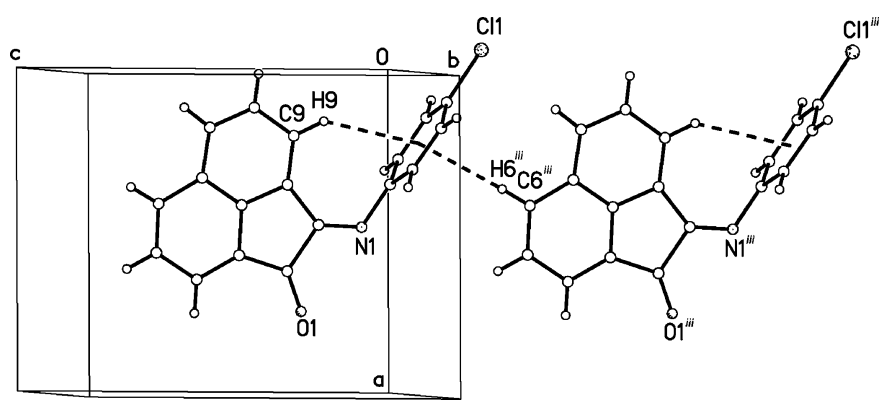

Figure 5

A view of the $\mathrm{C}-\mathrm{H} \cdots \pi$ interaction linking molecules together in the low temperature structure ( $\beta$-phase). A similar interaction occurs in the room-temperature structure ( $\alpha$-phase). Symmetry code: (iii) $x, y, z-1$.

\section{Synthesis and crystallization}

(E)-2-[(4-Chlorophenyl)imino]acenaphthylen-1-one (MIAN) was synthesized following a reported procedure (Kovach et al., 2011). Yellow block-like crystals were obtained by layering technical grade mixed hexanes over a solution of the compound in $\mathrm{CH}_{2} \mathrm{Cl}_{2}$.

\section{Refinement}

Crystal data, data collection and structure refinement details are summarized in Table 3. For both polymorphs, $\mathrm{H}$ atoms were included in calculated positions and treated as riding:
$\mathrm{C}-\mathrm{H}=0.94 \AA$ in the high temperature $\alpha$-phase and $0.95 \AA$ in the low temperature $\beta$-phase, with $U_{\text {iso }}(\mathrm{H})=1.2 U_{\text {eq }}(\mathrm{C})$.

\section{Acknowledgements}

The authors are grateful to Samantha Carrington for a sample of MIAN. LB thanks the China Scholarship Council for support of a joint $\mathrm{PhD}$ visit.

\section{References}

Bruker (2014). APEX2, SAINT, SADABS and TWINABS. BrukerNonius AXS Inc. Madison, Wisconsin, USA.

Carrington, S. J., Chakraborty, I. \& Mascharak, P. K. (2015). Dalton Trans. 44, 13828-13834.

Chakraborty, I., Carrington, S. J. \& Mascharak, P. K. (2014). Acc. Chem. Res. 47, 2603-2611.

Kovach, J., Peralta, M., Brennessel, W. W. \& Jones, W. D. (2011). J. Mol. Struct. 992, 33-38.

Macrae, C. F., Bruno, I. J., Chisholm, J. A., Edgington, P. R., McCabe, P., Pidcock, E., Rodriguez-Monge, L., Taylor, R., van de Streek, J. \& Wood, P. A. (2008). J. Appl. Cryst. 41, 466-470.

Sheldrick, G. M. (2008). Acta Cryst. A64, 112-122.

Sheldrick, G. M. (2015a). Acta Cryst. A71, 3-8.

Sheldrick, G. M. (2015b). Acta Cryst. C71, 3-8.

Stenger-Smith, J., Chakraborty, I., Carrington, S. \& Mascharak, P. (2017). Acta Cryst. C73, 357-361.

Takahashi, H. \& Ito, Y. (2010). CrystEngComm, 12, 1628-1634.

Takanabe, A., Katsufuji, T., Johmoto, K., Uekusa, H., Shiro, M., Koshima, H. \& Asahi, T. (2017). Crystals, 7, 7; doi:10.3390/ cryst7010007. 


\section{supporting information}

Acta Cryst. (2017). E73, 1255-1258 [https://doi.org/10.1107/S2056989017010659]

\section{A temperature-dependent phase transformation of $(E)-2-[(4-c h l o r o p h e n y l)-$ imino]acenaphthylen-1-one}

\section{Lipiao Bao and Marilyn M. Olmstead}

Computing details

For both structures, data collection: APEX2 (Bruker, 2014); cell refinement: SAINT (Bruker, 2014); data reduction: SAINT (Bruker, 2014); program(s) used to solve structure: SHELXT (Sheldrick, 2015a); program(s) used to refine structure: SHELXL2016/6 (Sheldrick, 2015b); molecular graphics: SHELXTL (Sheldrick, 2008) and Mercury (Macrae et al., 2008); software used to prepare material for publication: SHELXL2016/6 (Sheldrick, 2015b).

(E)-2-[(4-Chlorophenyl)imino]acenaphthylen-1-one (alpha)

\section{Crystal data}

$\mathrm{C}_{18} \mathrm{H}_{10} \mathrm{ClNO}$

$M_{r}=291.72$

Monoclinic, $P 2{ }_{1} / m$

$a=9.0447$ (12) $\AA$

$b=6.8764(9) \AA$

$c=10.9021(14) \AA$

$\beta=92.959(2)^{\circ}$

$V=677.15(15) \AA^{3}$

$Z=2$

\section{Data collection}

Bruker APEXII diffractometer

Radiation source: fine focus sealed tube Detector resolution: 8.3 pixels $\mathrm{mm}^{-1}$ $\omega$ scans

Absorption correction: multi-scan

(SADABS; Bruker, 2014)

$T_{\min }=0.684, T_{\max }=0.745$

Refinement

Refinement on $F^{2}$

Least-squares matrix: full

$R\left[F^{2}>2 \sigma\left(F^{2}\right)\right]=0.039$

$w R\left(F^{2}\right)=0.097$

$S=1.04$

1496 reflections

121 parameters

0 restraints

Primary atom site location: dual
$F(000)=300$

$D_{\mathrm{x}}=1.431 \mathrm{Mg} \mathrm{m}^{-3}$

Mo $K \alpha$ radiation, $\lambda=0.71073 \AA$

Cell parameters from 1908 reflections

$\theta=5.7-52.3^{\circ}$

$\mu=0.28 \mathrm{~mm}^{-1}$

$T=250 \mathrm{~K}$

Block, yellow

$0.30 \times 0.20 \times 0.20 \mathrm{~mm}$

5458 measured reflections

1496 independent reflections

1227 reflections with $I>2 \sigma(I)$

$R_{\text {int }}=0.022$

$\theta_{\max }=26.4^{\circ}, \theta_{\min }=2.3^{\circ}$

$h=-10 \rightarrow 11$

$k=-8 \rightarrow 8$

$l=-13 \rightarrow 13$

Secondary atom site location: difference Fourier map

Hydrogen site location: inferred from neighbouring sites

$\mathrm{H}$-atom parameters constrained

$w=1 /\left[\sigma^{2}\left(F_{\mathrm{o}}^{2}\right)+(0.037 P)^{2}+0.2756 P\right]$

where $P=\left(F_{\mathrm{o}}{ }^{2}+2 F_{\mathrm{c}}{ }^{2}\right) / 3$

$(\Delta / \sigma)_{\max }<0.001$

$\Delta \rho_{\max }=0.26 \mathrm{e} \AA^{-3}$

$\Delta \rho_{\min }=-0.38$ e $\AA^{-3}$ 


\section{Special details}

Geometry. All esds (except the esd in the dihedral angle between two 1.s. planes) are estimated using the full covariance matrix. The cell esds are taken into account individually in the estimation of esds in distances, angles and torsion angles; correlations between esds in cell parameters are only used when they are defined by crystal symmetry. An approximate (isotropic) treatment of cell esds is used for estimating esds involving l.s. planes.

Fractional atomic coordinates and isotropic or equivalent isotropic displacement parameters $\left(\AA^{2}\right)$

\begin{tabular}{lllll}
\hline & $x$ & $y$ & $z$ & $U_{\text {iso }} * / U_{\text {eq }}$ \\
\hline C11 & $-0.05474(8)$ & 0.250000 & $-0.19593(6)$ & $0.0739(3)$ \\
O1 & $0.75377(18)$ & 0.250000 & $0.29014(16)$ & $0.0547(5)$ \\
N1 & $0.4932(2)$ & 0.250000 & $0.12642(17)$ & $0.0442(5)$ \\
C1 & $0.4858(2)$ & 0.250000 & $0.2426(2)$ & $0.0366(5)$ \\
C2 & $0.6292(2)$ & 0.250000 & $0.3259(2)$ & $0.0395(5)$ \\
C3 & $0.5810(2)$ & 0.250000 & $0.4537(2)$ & $0.0366(5)$ \\
C4 & $0.6571(3)$ & 0.250000 & $0.5661(2)$ & $0.0440(6)$ \\
H4 & 0.761142 & 0.250000 & 0.571881 & $0.053^{*}$ \\
C5 & $0.5750(3)$ & 0.250000 & $0.6722(2)$ & $0.0475(6)$ \\
H5 & 0.626114 & 0.250000 & 0.749460 & $0.057^{*}$ \\
C6 & $0.4230(3)$ & 0.250000 & $0.6670(2)$ & $0.0473(6)$ \\
H6 & 0.372512 & 0.250000 & 0.740235 & $0.057^{*}$ \\
C7 & $0.1855(3)$ & 0.250000 & $0.5310(2)$ & $0.0564(7)$ \\
H7 & 0.124153 & 0.250000 & 0.598029 & $0.068^{*}$ \\
C8 & $0.1232(3)$ & 0.250000 & $0.4135(2)$ & $0.0584(7)$ \\
H8 & 0.019501 & 0.250000 & 0.402169 & $0.070^{*}$ \\
C9 & $0.2096(2)$ & 0.250000 & $0.3087(2)$ & $0.0455(6)$ \\
H9 & 0.164153 & 0.250000 & 0.229206 & $0.055^{*}$ \\
C10 & $0.3610(2)$ & 0.250000 & $0.32570(19)$ & $0.0361(5)$ \\
C11 & $0.4247(2)$ & 0.250000 & $0.44731(19)$ & $0.0347(5)$ \\
C12 & $0.3414(3)$ & 0.250000 & $0.5522(2)$ & $0.0419(5)$ \\
C13 & $0.3601(2)$ & 0.250000 & $0.05083(19)$ & $0.0398(5)$ \\
C14 & $0.29812(19)$ & $0.0762(3)$ & $0.00977(15)$ & $0.0479(4)$ \\
H14 & 0.342774 & -0.042208 & 0.033729 & $0.058^{*}$ \\
C15 & $0.17066(19)$ & $0.0757(3)$ & $-0.06642(15)$ & $0.0514(5)$ \\
H15 & 0.127910 & -0.042285 & -0.093524 & $0.062^{*}$ \\
C16 & $0.1076(3)$ & 0.250000 & $-0.1018(2)$ & $0.0471(6)$ \\
& & & &
\end{tabular}

Atomic displacement parameters $\left(\AA^{2}\right)$

\begin{tabular}{lllllll}
\hline & $U^{11}$ & $U^{22}$ & $U^{33}$ & $U^{12}$ & $U^{13}$ & $U^{23}$ \\
\hline C11 & $0.0487(4)$ & $0.1311(8)$ & $0.0411(4)$ & 0.000 & $-0.0069(3)$ & 0.000 \\
O1 & $0.0345(9)$ & $0.0793(13)$ & $0.0506(10)$ & 0.000 & $0.0053(7)$ & 0.000 \\
N1 & $0.0405(10)$ & $0.0580(13)$ & $0.0342(10)$ & 0.000 & $0.0025(8)$ & 0.000 \\
C1 & $0.0366(11)$ & $0.0376(12)$ & $0.0357(12)$ & 0.000 & $0.0026(9)$ & 0.000 \\
C2 & $0.0357(12)$ & $0.0408(13)$ & $0.0420(12)$ & 0.000 & $0.0007(9)$ & 0.000 \\
C3 & $0.0395(12)$ & $0.0338(11)$ & $0.0364(11)$ & 0.000 & $-0.0005(9)$ & 0.000 \\
C4 & $0.0430(13)$ & $0.0447(14)$ & $0.0433(13)$ & 0.000 & $-0.0084(10)$ & 0.000 \\
C5 & $0.0613(16)$ & $0.0465(14)$ & $0.0335(12)$ & 0.000 & $-0.0088(11)$ & 0.000
\end{tabular}




\begin{tabular}{lllllll} 
C6 & $0.0611(16)$ & $0.0473(14)$ & $0.0337(12)$ & 0.000 & $0.0060(11)$ & 0.000 \\
C7 & $0.0450(14)$ & $0.081(2)$ & $0.0442(14)$ & 0.000 & $0.0130(11)$ & 0.000 \\
C8 & $0.0350(12)$ & $0.089(2)$ & $0.0517(15)$ & 0.000 & $0.0075(11)$ & 0.000 \\
C9 & $0.0361(12)$ & $0.0619(16)$ & $0.0383(12)$ & 0.000 & $-0.0010(9)$ & 0.000 \\
C10 & $0.0369(11)$ & $0.0374(12)$ & $0.0340(11)$ & 0.000 & $0.0025(9)$ & 0.000 \\
C11 & $0.0379(11)$ & $0.0312(11)$ & $0.0350(11)$ & 0.000 & $0.0005(9)$ & 0.000 \\
C12 & $0.0458(13)$ & $0.0424(13)$ & $0.0377(12)$ & 0.000 & $0.0056(10)$ & 0.000 \\
C13 & $0.0396(12)$ & $0.0523(14)$ & $0.0280(10)$ & 0.000 & $0.0072(9)$ & 0.000 \\
C14 & $0.0494(9)$ & $0.0480(10)$ & $0.0465(9)$ & $0.0010(8)$ & $0.0034(7)$ & $0.0022(8)$ \\
C15 & $0.0499(10)$ & $0.0593(12)$ & $0.0452(9)$ & $-0.0073(9)$ & $0.0038(8)$ & $-0.0108(9)$ \\
C16 & $0.0401(12)$ & $0.0743(18)$ & $0.0269(11)$ & 0.000 & $0.0028(9)$ & 0.000 \\
\hline
\end{tabular}

Geometric parameters $\left(\AA,{ }^{\circ}\right)$

\begin{tabular}{|c|c|c|c|}
\hline $\mathrm{C} 11-\mathrm{C} 16$ & $1.747(2)$ & $\mathrm{C} 7-\mathrm{C} 8$ & $1.372(4)$ \\
\hline $\mathrm{O} 1-\mathrm{C} 2$ & $1.211(3)$ & $\mathrm{C} 7-\mathrm{C} 12$ & $1.417(3)$ \\
\hline $\mathrm{N} 1-\mathrm{C} 1$ & $1.272(3)$ & $\mathrm{C} 7-\mathrm{H} 7$ & 0.9400 \\
\hline $\mathrm{N} 1-\mathrm{C} 13$ & $1.423(3)$ & $\mathrm{C} 8-\mathrm{C} 9$ & $1.417(3)$ \\
\hline $\mathrm{C} 1-\mathrm{C} 10$ & $1.484(3)$ & $\mathrm{C} 8-\mathrm{H} 8$ & 0.9400 \\
\hline $\mathrm{C} 1-\mathrm{C} 2$ & $1.545(3)$ & $\mathrm{C} 9-\mathrm{C} 10$ & $1.372(3)$ \\
\hline $\mathrm{C} 2-\mathrm{C} 3$ & $1.481(3)$ & $\mathrm{C} 9-\mathrm{H} 9$ & 0.9400 \\
\hline $\mathrm{C} 3-\mathrm{C} 4$ & $1.373(3)$ & $\mathrm{C} 10-\mathrm{C} 11$ & $1.418(3)$ \\
\hline $\mathrm{C} 3-\mathrm{C} 11$ & $1.413(3)$ & $\mathrm{C} 11-\mathrm{C} 12$ & $1.402(3)$ \\
\hline $\mathrm{C} 4-\mathrm{C} 5$ & $1.406(3)$ & $\mathrm{C} 13-\mathrm{C} 14$ & $1.385(2)$ \\
\hline $\mathrm{C} 4-\mathrm{H} 4$ & 0.9400 & $\mathrm{C} 13-\mathrm{C} 14^{\mathrm{i}}$ & $1.385(2)$ \\
\hline $\mathrm{C} 5-\mathrm{C} 6$ & $1.373(4)$ & $\mathrm{C} 14-\mathrm{C} 15$ & $1.386(2)$ \\
\hline $\mathrm{C} 5-\mathrm{H} 5$ & 0.9400 & $\mathrm{C} 14-\mathrm{H} 14$ & 0.9400 \\
\hline $\mathrm{C} 6-\mathrm{C} 12$ & $1.420(3)$ & $\mathrm{C} 15-\mathrm{C} 16$ & $1.374(2)$ \\
\hline C6-H6 & 0.9400 & $\mathrm{C} 15-\mathrm{H} 15$ & 0.9400 \\
\hline $\mathrm{C} 1-\mathrm{N} 1-\mathrm{C} 13$ & $119.39(19)$ & $\mathrm{C} 10-\mathrm{C} 9-\mathrm{C} 8$ & $118.6(2)$ \\
\hline $\mathrm{N} 1-\mathrm{C} 1-\mathrm{C} 10$ & $133.5(2)$ & $\mathrm{C} 10-\mathrm{C} 9-\mathrm{H} 9$ & 120.7 \\
\hline $\mathrm{N} 1-\mathrm{C} 1-\mathrm{C} 2$ & $120.02(19)$ & $\mathrm{C} 8-\mathrm{C} 9-\mathrm{H} 9$ & 120.7 \\
\hline $\mathrm{C} 10-\mathrm{C} 1-\mathrm{C} 2$ & $106.45(17)$ & $\mathrm{C} 9-\mathrm{C} 10-\mathrm{C} 11$ & $118.7(2)$ \\
\hline $\mathrm{O} 1-\mathrm{C} 2-\mathrm{C} 3$ & $128.8(2)$ & $\mathrm{C} 9-\mathrm{C} 10-\mathrm{C} 1$ & $134.7(2)$ \\
\hline $\mathrm{O} 1-\mathrm{C} 2-\mathrm{C} 1$ & $125.3(2)$ & $\mathrm{C} 11-\mathrm{C} 10-\mathrm{C} 1$ & $106.63(18)$ \\
\hline $\mathrm{C} 3-\mathrm{C} 2-\mathrm{C} 1$ & $105.91(18)$ & $\mathrm{C} 12-\mathrm{C} 11-\mathrm{C} 3$ & $122.6(2)$ \\
\hline $\mathrm{C} 4-\mathrm{C} 3-\mathrm{C} 11$ & $119.9(2)$ & $\mathrm{C} 12-\mathrm{C} 11-\mathrm{C} 10$ & $123.6(2)$ \\
\hline $\mathrm{C} 4-\mathrm{C} 3-\mathrm{C} 2$ & $132.9(2)$ & $\mathrm{C} 3-\mathrm{C} 11-\mathrm{C} 10$ & $113.79(19)$ \\
\hline $\mathrm{C} 11-\mathrm{C} 3-\mathrm{C} 2$ & $107.22(19)$ & $\mathrm{C} 11-\mathrm{C} 12-\mathrm{C} 7$ & $116.0(2)$ \\
\hline $\mathrm{C} 3-\mathrm{C} 4-\mathrm{C} 5$ & $118.2(2)$ & $\mathrm{C} 11-\mathrm{C} 12-\mathrm{C} 6$ & $116.3(2)$ \\
\hline $\mathrm{C} 3-\mathrm{C} 4-\mathrm{H} 4$ & 120.9 & $\mathrm{C} 7-\mathrm{C} 12-\mathrm{C} 6$ & $127.7(2)$ \\
\hline $\mathrm{C} 5-\mathrm{C} 4-\mathrm{H} 4$ & 120.9 & $\mathrm{C} 14-\mathrm{C} 13-\mathrm{C} 14^{\mathrm{i}}$ & $119.3(2)$ \\
\hline $\mathrm{C} 6-\mathrm{C} 5-\mathrm{C} 4$ & $122.4(2)$ & $\mathrm{C} 14-\mathrm{C} 13-\mathrm{N} 1$ & $120.24(11)$ \\
\hline $\mathrm{C} 6-\mathrm{C} 5-\mathrm{H} 5$ & 118.8 & $\mathrm{C} 14-\mathrm{C} 13-\mathrm{N} 1$ & $120.24(11)$ \\
\hline $\mathrm{C} 4-\mathrm{C} 5-\mathrm{H} 5$ & 118.8 & $\mathrm{C} 13-\mathrm{C} 14-\mathrm{C} 15$ & $120.43(18)$ \\
\hline $\mathrm{C} 5-\mathrm{C} 6-\mathrm{C} 12$ & $120.7(2)$ & $\mathrm{C} 13-\mathrm{C} 14-\mathrm{H} 14$ & 119.8 \\
\hline $\mathrm{C} 5-\mathrm{C} 6-\mathrm{H} 6$ & 119.7 & $\mathrm{C} 15-\mathrm{C} 14-\mathrm{H} 14$ & 119.8 \\
\hline
\end{tabular}




\begin{tabular}{|c|c|c|c|}
\hline $\mathrm{C} 12-\mathrm{C} 6-\mathrm{H} 6$ & 119.7 & $\mathrm{C} 16-\mathrm{C} 15-\mathrm{C} 14$ & $119.13(18)$ \\
\hline $\mathrm{C} 8-\mathrm{C} 7-\mathrm{C} 12$ & $120.6(2)$ & $\mathrm{C} 16-\mathrm{C} 15-\mathrm{H} 15$ & 120.4 \\
\hline $\mathrm{C} 8-\mathrm{C} 7-\mathrm{H} 7$ & 119.7 & $\mathrm{C} 14-\mathrm{C} 15-\mathrm{H} 15$ & 120.4 \\
\hline $\mathrm{C} 12-\mathrm{C} 7-\mathrm{H} 7$ & 119.7 & $\mathrm{C} 15^{\mathrm{i}}-\mathrm{C} 16-\mathrm{C} 15$ & $121.4(2)$ \\
\hline $\mathrm{C} 7-\mathrm{C} 8-\mathrm{C} 9$ & $122.4(2)$ & $\mathrm{C} 15^{\mathrm{i}}-\mathrm{C} 16-\mathrm{Cl1}$ & $119.28(11)$ \\
\hline $\mathrm{C} 7-\mathrm{C} 8-\mathrm{H} 8$ & 118.8 & $\mathrm{C} 15-\mathrm{C} 16-\mathrm{Cl1}$ & $119.28(11)$ \\
\hline $\mathrm{C} 9-\mathrm{C} 8-\mathrm{H} 8$ & 118.8 & & \\
\hline $\mathrm{C} 13-\mathrm{N} 1-\mathrm{C} 1-\mathrm{C} 10$ & $0.000(1)$ & $\mathrm{C} 2-\mathrm{C} 3-\mathrm{C} 11-\mathrm{C} 12$ & $180.000(1)$ \\
\hline $\mathrm{C} 13-\mathrm{N} 1-\mathrm{C} 1-\mathrm{C} 2$ & $180.000(1)$ & $\mathrm{C} 4-\mathrm{C} 3-\mathrm{C} 11-\mathrm{C} 10$ & $180.000(1)$ \\
\hline $\mathrm{N} 1-\mathrm{C} 1-\mathrm{C} 2-\mathrm{O} 1$ & $0.000(1)$ & $\mathrm{C} 2-\mathrm{C} 3-\mathrm{C} 11-\mathrm{C} 10$ & $0.000(1)$ \\
\hline $\mathrm{C} 10-\mathrm{C} 1-\mathrm{C} 2-\mathrm{O} 1$ & $180.000(1)$ & $\mathrm{C} 9-\mathrm{C} 10-\mathrm{C} 11-\mathrm{C} 12$ & $0.000(1)$ \\
\hline $\mathrm{N} 1-\mathrm{C} 1-\mathrm{C} 2-\mathrm{C} 3$ & $180.000(1)$ & $\mathrm{C} 1-\mathrm{C} 10-\mathrm{C} 11-\mathrm{C} 12$ & $180.000(1)$ \\
\hline $\mathrm{C} 10-\mathrm{C} 1-\mathrm{C} 2-\mathrm{C} 3$ & $0.000(1)$ & $\mathrm{C} 9-\mathrm{C} 10-\mathrm{C} 11-\mathrm{C} 3$ & $180.000(1)$ \\
\hline $\mathrm{O} 1-\mathrm{C} 2-\mathrm{C} 3-\mathrm{C} 4$ & $0.000(1)$ & $\mathrm{C} 1-\mathrm{C} 10-\mathrm{C} 11-\mathrm{C} 3$ & $0.000(1)$ \\
\hline $\mathrm{C} 1-\mathrm{C} 2-\mathrm{C} 3-\mathrm{C} 4$ & $180.000(1)$ & $\mathrm{C} 3-\mathrm{C} 11-\mathrm{C} 12-\mathrm{C} 7$ & $180.000(1)$ \\
\hline $\mathrm{O} 1-\mathrm{C} 2-\mathrm{C} 3-\mathrm{C} 11$ & $180.000(1)$ & $\mathrm{C} 10-\mathrm{C} 11-\mathrm{C} 12-\mathrm{C} 7$ & $0.000(1)$ \\
\hline $\mathrm{C} 1-\mathrm{C} 2-\mathrm{C} 3-\mathrm{C} 11$ & $0.000(1)$ & $\mathrm{C} 3-\mathrm{C} 11-\mathrm{C} 12-\mathrm{C} 6$ & $0.000(1)$ \\
\hline $\mathrm{C} 11-\mathrm{C} 3-\mathrm{C} 4-\mathrm{C} 5$ & $0.000(1)$ & $\mathrm{C} 10-\mathrm{C} 11-\mathrm{C} 12-\mathrm{C} 6$ & $180.000(1)$ \\
\hline $\mathrm{C} 2-\mathrm{C} 3-\mathrm{C} 4-\mathrm{C} 5$ & $180.000(1)$ & $\mathrm{C} 8-\mathrm{C} 7-\mathrm{C} 12-\mathrm{C} 11$ & $0.000(1)$ \\
\hline $\mathrm{C} 3-\mathrm{C} 4-\mathrm{C} 5-\mathrm{C} 6$ & $0.000(1)$ & $\mathrm{C} 8-\mathrm{C} 7-\mathrm{C} 12-\mathrm{C} 6$ & $180.000(1)$ \\
\hline $\mathrm{C} 4-\mathrm{C} 5-\mathrm{C} 6-\mathrm{C} 12$ & $0.000(1)$ & $\mathrm{C} 5-\mathrm{C} 6-\mathrm{C} 12-\mathrm{C} 11$ & $0.000(1)$ \\
\hline $\mathrm{C} 12-\mathrm{C} 7-\mathrm{C} 8-\mathrm{C} 9$ & $0.000(1)$ & $\mathrm{C} 5-\mathrm{C} 6-\mathrm{C} 12-\mathrm{C} 7$ & $180.000(1)$ \\
\hline $\mathrm{C} 7-\mathrm{C} 8-\mathrm{C} 9-\mathrm{C} 10$ & $0.000(1)$ & $\mathrm{C} 1-\mathrm{N} 1-\mathrm{C} 13-\mathrm{C} 14$ & $-92.57(18)$ \\
\hline $\mathrm{C} 8-\mathrm{C} 9-\mathrm{C} 10-\mathrm{C} 11$ & $0.000(1)$ & $\mathrm{C} 1-\mathrm{N} 1-\mathrm{C} 13-\mathrm{C} 14^{\mathrm{i}}$ & $92.57(18)$ \\
\hline $\mathrm{C} 8-\mathrm{C} 9-\mathrm{C} 10-\mathrm{C} 1$ & $180.000(1)$ & $\mathrm{C} 14-\mathrm{C} 13-\mathrm{C} 14-\mathrm{C} 15$ & $-3.4(3)$ \\
\hline $\mathrm{N} 1-\mathrm{C} 1-\mathrm{C} 10-\mathrm{C} 9$ & $0.000(1)$ & $\mathrm{N} 1-\mathrm{C} 13-\mathrm{C} 14-\mathrm{C} 15$ & $-178.35(17)$ \\
\hline $\mathrm{C} 2-\mathrm{C} 1-\mathrm{C} 10-\mathrm{C} 9$ & $180.000(1)$ & $\mathrm{C} 13-\mathrm{C} 14-\mathrm{C} 15-\mathrm{C} 16$ & $0.7(3)$ \\
\hline $\mathrm{N} 1-\mathrm{C} 1-\mathrm{C} 10-\mathrm{C} 11$ & $180.000(1)$ & $\mathrm{C} 14-\mathrm{C} 15-\mathrm{C} 16-\mathrm{C} 15^{\mathrm{i}}$ & $2.0(3)$ \\
\hline $\mathrm{C} 2-\mathrm{C} 1-\mathrm{C} 10-\mathrm{C} 11$ & $0.000(1)$ & $\mathrm{C} 14-\mathrm{C} 15-\mathrm{C} 16-\mathrm{Cl1}$ & $-179.01(14)$ \\
\hline $\mathrm{C} 4-\mathrm{C} 3-\mathrm{C} 11-\mathrm{C} 12$ & $0.000(1)$ & & \\
\hline
\end{tabular}

Symmetry code: (i) $x,-y+1 / 2, z$.

Hydrogen-bond geometry $(\AA, \stackrel{\circ}{)}$

$\mathrm{Cg}$ is the centroid of the 4-chlorophenyl ring (C13-C16/C14A/C15A).

\begin{tabular}{lllll}
\hline$D-\mathrm{H} \cdots A$ & $D-\mathrm{H}$ & $\mathrm{H} \cdots A$ & $D \cdots A$ & $D-\mathrm{H} \cdots A$ \\
\hline $\mathrm{C} 6-\mathrm{H} 6 \cdots C g^{\mathrm{ii}}$ & 0.94 & 2.86 & $3.803(2)$ & 177 \\
$\mathrm{C} 9-\mathrm{H} 9 \cdots C g$ & 0.94 & 2.88 & $3.668(11)$ & 128
\end{tabular}

Symmetry code: (ii) $x+1, y, z+1$.

(E)-2-[(4-Chlorophenyl)imino]acenaphthylen-1-one (beta)

Crystal data

$\mathrm{C}_{18} \mathrm{H}_{10} \mathrm{CINO}$

$M_{r}=291.72$

Triclinic, $P \overline{1}$

$a=9.0764$ (10) $\AA$

$$
\begin{aligned}
& b=6.8187(8) \AA \\
& c=10.7450(12) \AA \\
& \alpha=90.880(2)^{\circ} \\
& \beta=92.780(2)^{\circ}
\end{aligned}
$$


$\gamma=96.259(2)^{\circ}$

$V=660.12(13) \AA^{3}$

$Z=2$

$F(000)=300$

$D_{\mathrm{x}}=1.468 \mathrm{Mg} \mathrm{m}^{-3}$

Mo $K \alpha$ radiation, $\lambda=0.71073 \AA$

Data collection

Bruker APEXII

diffractometer

Radiation source: fine focus sealed tube

Detector resolution: 8.3 pixels $\mathrm{mm}^{-1}$

$\omega$ scans

Absorption correction: multi-scan

(TWINABS; Bruker, 2014)

$T_{\text {min }}=0.629, T_{\max }=0.746$

Refinement

Refinement on $F^{2}$

Least-squares matrix: full

$R\left[F^{2}>2 \sigma\left(F^{2}\right)\right]=0.031$

$w R\left(F^{2}\right)=0.092$

$S=1.08$

2949 reflections

193 parameters

0 restraints

Primary atom site location: dual
Cell parameters from 9928 reflections

$\theta=4.5-55.2^{\circ}$

$\mu=0.29 \mathrm{~mm}^{-1}$

$T=90 \mathrm{~K}$

Block, yellow

$0.30 \times 0.20 \times 0.20 \mathrm{~mm}$

34083 measured reflections

2949 independent reflections

2726 reflections with $I>2 \sigma(I)$

$R_{\text {int }}=0.023$

$\theta_{\max }=27.6^{\circ}, \theta_{\min }=1.9^{\circ}$

$h=-11 \rightarrow 11$

$k=-8 \rightarrow 8$

$l=-13 \rightarrow 13$

Secondary atom site location: difference Fourier map

Hydrogen site location: inferred from neighbouring sites

$\mathrm{H}$-atom parameters constrained

$w=1 /\left[\sigma^{2}\left(F_{\mathrm{o}}^{2}\right)+(0.0535 P)^{2}+0.1518 P\right]$

where $P=\left(F_{\mathrm{o}}^{2}+2 F_{\mathrm{c}}^{2}\right) / 3$

$(\Delta / \sigma)_{\max }<0.001$

$\Delta \rho_{\max }=0.33$ e $\AA^{-3}$

$\Delta \rho_{\min }=-0.24$ e $\AA^{-3}$

\section{Special details}

Geometry. All esds (except the esd in the dihedral angle between two 1.s. planes) are estimated using the full covariance matrix. The cell esds are taken into account individually in the estimation of esds in distances, angles and torsion angles; correlations between esds in cell parameters are only used when they are defined by crystal symmetry. An approximate (isotropic) treatment of cell esds is used for estimating esds involving l.s. planes.

Refinement. Refined as a 4-component twin.

Fractional atomic coordinates and isotropic or equivalent isotropic displacement parameters $\left(\AA^{2}\right)$

\begin{tabular}{lllll}
\hline & $x$ & $y$ & $z$ & $U_{\text {iso }} * / U_{\text {eq }}$ \\
\hline C11 & $-0.06705(4)$ & $0.27235(6)$ & $-0.20019(3)$ & $0.02295(12)$ \\
O1 & $0.74526(11)$ & $0.24307(17)$ & $0.28273(9)$ & $0.0189(2)$ \\
N1 & $0.48366(13)$ & $0.23262(19)$ & $0.11662(11)$ & $0.0160(3)$ \\
C1 & $0.47710(15)$ & $0.2430(2)$ & $0.23456(13)$ & $0.0132(3)$ \\
C2 & $0.62087(15)$ & $0.2429(2)$ & $0.31934(13)$ & $0.0138(3)$ \\
C3 & $0.57248(15)$ & $0.2463(2)$ & $0.44935(13)$ & $0.0136(3)$ \\
C4 & $0.64841(16)$ & $0.2421(2)$ & $0.56328(13)$ & $0.0160(3)$ \\
H4 & 0.752714 & 0.237152 & 0.568827 & $0.019^{*}$ \\
C5 & $0.56597(16)$ & $0.2455(2)$ & $0.67222(13)$ & $0.0172(3)$ \\
H5 & 0.616929 & 0.242653 & 0.751402 & $0.021^{*}$ \\
C6 & $0.41399(16)$ & $0.2527(2)$ & $0.66699(13)$ & $0.0171(3)$ \\
H6 & 0.363047 & 0.256111 & 0.742037 & $0.021^{*}$ \\
C7 & $0.17740(16)$ & $0.2579(2)$ & $0.52922(14)$ & $0.0200(3)$
\end{tabular}




$\begin{array}{lllll}\text { H7 } & 0.115871 & 0.260723 & 0.598122 & 0.024^{*} \\ \text { C8 } & 0.11510(16) & 0.2563(3) & 0.40886(14) & 0.0212(3) \\ \text { H8 } & 0.010792 & 0.257525 & 0.397112 & 0.025^{*} \\ \text { C9 } & 0.20184(15) & 0.2531(2) & 0.30235(13) & 0.0165(3) \\ \text { H9 } & 0.156281 & 0.252026 & 0.220822 & 0.020^{*} \\ \text { C10 } & 0.35329(15) & 0.2514(2) & 0.31941(12) & 0.0136(3) \\ \text { C11 } & 0.41694(15) & 0.2523(2) & 0.44327(13) & 0.0132(3) \\ \text { C12 } & 0.33329(16) & 0.2552(2) & 0.55003(13) & 0.0156(3) \\ \text { C13 } & 0.35083(15) & 0.2411(2) & 0.04076(12) & 0.0148(3) \\ \text { C14 } & 0.27646(16) & 0.0687(2) & -0.01417(13) & 0.0173(3) \\ \text { H14 } & 0.313778 & -0.054584 & -0.000952 & 0.021^{*} \\ \text { C15 } & 0.14723(16) & 0.0784(2) & -0.08846(14) & 0.0184(3) \\ \text { H15 } & 0.094740 & -0.038308 & -0.124808 & 0.022^{*} \\ \text { C16 } & 0.09615(15) & 0.2603(2) & -0.10873(12) & 0.0167(3) \\ \text { C17 } & 0.17291(16) & 0.4341(2) & -0.05980(13) & 0.0178(3) \\ \text { H17 } & 0.138749 & 0.558005 & -0.077520 & 0.021^{*} \\ \text { C18 } & 0.30080(16) & 0.4234(2) & 0.01565(13) & 0.0170(3) \\ \text { H18 } & 0.354230 & 0.540777 & 0.050193 & 0.020^{*}\end{array}$

Atomic displacement parameters $\left(\AA^{2}\right)$

\begin{tabular}{lllllll}
\hline & $U^{11}$ & $U^{22}$ & $U^{33}$ & $U^{12}$ & $U^{13}$ & $U^{23}$ \\
\hline C11 & $0.01595(18)$ & $0.0391(2)$ & $0.01433(17)$ & $0.00712(15)$ & $-0.00253(11)$ & $-0.00184(15)$ \\
O1 & $0.0136(5)$ & $0.0254(6)$ & $0.0180(5)$ & $0.0036(4)$ & $0.0023(4)$ & $0.0018(4)$ \\
N1 & $0.0151(6)$ & $0.0200(6)$ & $0.0133(5)$ & $0.0039(5)$ & $-0.0001(4)$ & $0.0016(5)$ \\
C1 & $0.0122(6)$ & $0.0124(6)$ & $0.0152(6)$ & $0.0022(5)$ & $0.0006(5)$ & $0.0013(5)$ \\
C2 & $0.0146(6)$ & $0.0129(7)$ & $0.0139(6)$ & $0.0023(5)$ & $-0.0007(5)$ & $0.0012(5)$ \\
C3 & $0.0153(7)$ & $0.0105(7)$ & $0.0148(6)$ & $0.0011(5)$ & $0.0006(5)$ & $0.0000(5)$ \\
C4 & $0.0166(7)$ & $0.0153(7)$ & $0.0159(6)$ & $0.0022(5)$ & $-0.0012(5)$ & $0.0007(5)$ \\
C5 & $0.0232(7)$ & $0.0160(7)$ & $0.0123(6)$ & $0.0023(5)$ & $-0.0024(5)$ & $0.0008(5)$ \\
C6 & $0.0226(7)$ & $0.0163(7)$ & $0.0125(6)$ & $0.0008(5)$ & $0.0034(5)$ & $0.0006(5)$ \\
C7 & $0.0173(7)$ & $0.0261(8)$ & $0.0167(7)$ & $0.0011(6)$ & $0.0051(5)$ & $0.0005(6)$ \\
C8 & $0.0124(6)$ & $0.0307(9)$ & $0.0207(7)$ & $0.0022(6)$ & $0.0026(5)$ & $0.0019(6)$ \\
C9 & $0.0154(7)$ & $0.0203(7)$ & $0.0136(6)$ & $0.0011(5)$ & $-0.0002(5)$ & $0.0009(5)$ \\
C10 & $0.0159(7)$ & $0.0122(7)$ & $0.0128(6)$ & $0.0013(5)$ & $0.0019(5)$ & $0.0011(5)$ \\
C11 & $0.0151(6)$ & $0.0104(6)$ & $0.0140(6)$ & $0.0007(5)$ & $0.0006(5)$ & $0.0012(5)$ \\
C12 & $0.0185(7)$ & $0.0140(7)$ & $0.0140(6)$ & $0.0007(5)$ & $0.0015(5)$ & $0.0007(5)$ \\
C13 & $0.0129(6)$ & $0.0225(8)$ & $0.0098(6)$ & $0.0035(5)$ & $0.0024(5)$ & $0.0019(5)$ \\
C14 & $0.0181(7)$ & $0.0189(7)$ & $0.0157(7)$ & $0.0047(5)$ & $0.0036(5)$ & $0.0014(6)$ \\
C15 & $0.0157(7)$ & $0.0220(8)$ & $0.0173(7)$ & $0.0014(5)$ & $0.0020(5)$ & $-0.0035(6)$ \\
C16 & $0.0123(6)$ & $0.0287(8)$ & $0.0097(6)$ & $0.0046(6)$ & $0.0011(5)$ & $0.0015(6)$ \\
C17 & $0.0191(7)$ & $0.0211(8)$ & $0.0144(6)$ & $0.0066(6)$ & $0.0023(5)$ & $0.0024(6)$ \\
C18 & $0.0180(7)$ & $0.0181(7)$ & $0.0149(6)$ & $0.0025(5)$ & $0.0006(5)$ & $-0.0011(5)$ \\
& & & & & &
\end{tabular}

Geometric parameters $(\AA, \stackrel{\circ}{)})$

\begin{tabular}{llll}
\hline $\mathrm{C} 11-\mathrm{C} 16$ & $1.7478(14)$ & $\mathrm{C} 7-\mathrm{H} 7$ & 0.9500 \\
$\mathrm{O} 1-\mathrm{C} 2$ & $1.2133(17)$ & $\mathrm{C} 8-\mathrm{C} 9$ & $1.4214(19)$
\end{tabular}




\begin{tabular}{|c|c|c|c|}
\hline $\mathrm{N} 1-\mathrm{C} 1$ & $1.2728(18)$ & $\mathrm{C} 8-\mathrm{H} 8$ & 0.9500 \\
\hline $\mathrm{N} 1-\mathrm{C} 13$ & $1.4292(17)$ & $\mathrm{C} 9-\mathrm{C} 10$ & $1.3793(19)$ \\
\hline $\mathrm{C} 1-\mathrm{C} 10$ & $1.4863(18)$ & C9-H9 & 0.9500 \\
\hline $\mathrm{C} 1-\mathrm{C} 2$ & $1.5548(18)$ & $\mathrm{C} 10-\mathrm{C} 11$ & $1.4246(18)$ \\
\hline $\mathrm{C} 2-\mathrm{C} 3$ & $1.4851(19)$ & $\mathrm{C} 11-\mathrm{C} 12$ & $1.4070(19)$ \\
\hline $\mathrm{C} 3-\mathrm{C} 4$ & $1.3777(19)$ & $\mathrm{C} 13-\mathrm{C} 18$ & $1.394(2)$ \\
\hline $\mathrm{C} 3-\mathrm{C} 11$ & $1.4151(19)$ & $\mathrm{C} 13-\mathrm{C} 14$ & $1.398(2)$ \\
\hline $\mathrm{C} 4-\mathrm{C} 5$ & $1.421(2)$ & $\mathrm{C} 14-\mathrm{C} 15$ & $1.395(2)$ \\
\hline $\mathrm{C} 4-\mathrm{H} 4$ & 0.9500 & $\mathrm{C} 14-\mathrm{H} 14$ & 0.9500 \\
\hline $\mathrm{C} 5-\mathrm{C} 6$ & $1.384(2)$ & $\mathrm{C} 15-\mathrm{C} 16$ & $1.387(2)$ \\
\hline $\mathrm{C} 5-\mathrm{H} 5$ & 0.9500 & $\mathrm{C} 15-\mathrm{H} 15$ & 0.9500 \\
\hline $\mathrm{C} 6-\mathrm{C} 12$ & $1.4249(19)$ & $\mathrm{C} 16-\mathrm{C} 17$ & $1.390(2)$ \\
\hline $\mathrm{C} 6-\mathrm{H} 6$ & 0.9500 & $\mathrm{C} 17-\mathrm{C} 18$ & $1.393(2)$ \\
\hline $\mathrm{C} 7-\mathrm{C} 8$ & $1.385(2)$ & C17-H17 & 0.9500 \\
\hline $\mathrm{C} 7-\mathrm{C} 12$ & $1.424(2)$ & $\mathrm{C} 18-\mathrm{H} 18$ & 0.9500 \\
\hline $\mathrm{C} 1-\mathrm{N} 1-\mathrm{C} 13$ & $118.79(12)$ & $\mathrm{C} 9-\mathrm{C} 10-\mathrm{C} 11$ & $118.72(12)$ \\
\hline $\mathrm{N} 1-\mathrm{C} 1-\mathrm{C} 10$ & $133.62(13)$ & $\mathrm{C} 9-\mathrm{C} 10-\mathrm{C} 1$ & $134.58(12)$ \\
\hline $\mathrm{N} 1-\mathrm{C} 1-\mathrm{C} 2$ & $119.95(12)$ & $\mathrm{C} 11-\mathrm{C} 10-\mathrm{C} 1$ & $106.67(11)$ \\
\hline $\mathrm{C} 10-\mathrm{C} 1-\mathrm{C} 2$ & $106.41(11)$ & $\mathrm{C} 12-\mathrm{C} 11-\mathrm{C} 3$ & $122.84(13)$ \\
\hline $\mathrm{O} 1-\mathrm{C} 2-\mathrm{C} 3$ & $128.96(13)$ & $\mathrm{C} 12-\mathrm{C} 11-\mathrm{C} 10$ & $123.41(13)$ \\
\hline $\mathrm{O} 1-\mathrm{C} 2-\mathrm{C} 1$ & $125.29(12)$ & $\mathrm{C} 3-\mathrm{C} 11-\mathrm{C} 10$ & $113.74(12)$ \\
\hline $\mathrm{C} 3-\mathrm{C} 2-\mathrm{C} 1$ & $105.75(11)$ & $\mathrm{C} 11-\mathrm{C} 12-\mathrm{C} 7$ & $116.47(13)$ \\
\hline $\mathrm{C} 4-\mathrm{C} 3-\mathrm{C} 11$ & $120.05(13)$ & $\mathrm{C} 11-\mathrm{C} 12-\mathrm{C} 6$ & $116.28(13)$ \\
\hline $\mathrm{C} 4-\mathrm{C} 3-\mathrm{C} 2$ & $132.53(13)$ & $\mathrm{C} 7-\mathrm{C} 12-\mathrm{C} 6$ & $127.25(13)$ \\
\hline $\mathrm{C} 11-\mathrm{C} 3-\mathrm{C} 2$ & $107.41(12)$ & $\mathrm{C} 18-\mathrm{C} 13-\mathrm{C} 14$ & $120.11(13)$ \\
\hline $\mathrm{C} 3-\mathrm{C} 4-\mathrm{C} 5$ & $117.97(13)$ & $\mathrm{C} 18-\mathrm{C} 13-\mathrm{N} 1$ & $119.71(13)$ \\
\hline $\mathrm{C} 3-\mathrm{C} 4-\mathrm{H} 4$ & 121.0 & $\mathrm{C} 14-\mathrm{C} 13-\mathrm{N} 1$ & $120.07(13)$ \\
\hline $\mathrm{C} 5-\mathrm{C} 4-\mathrm{H} 4$ & 121.0 & $\mathrm{C} 15-\mathrm{C} 14-\mathrm{C} 13$ & $119.71(14)$ \\
\hline $\mathrm{C} 6-\mathrm{C} 5-\mathrm{C} 4$ & $122.29(13)$ & $\mathrm{C} 15-\mathrm{C} 14-\mathrm{H} 14$ & 120.1 \\
\hline $\mathrm{C} 6-\mathrm{C} 5-\mathrm{H} 5$ & 118.9 & $\mathrm{C} 13-\mathrm{C} 14-\mathrm{H} 14$ & 120.1 \\
\hline $\mathrm{C} 4-\mathrm{C} 5-\mathrm{H} 5$ & 118.9 & $\mathrm{C} 16-\mathrm{C} 15-\mathrm{C} 14$ & $119.29(14)$ \\
\hline $\mathrm{C} 5-\mathrm{C} 6-\mathrm{C} 12$ & $120.57(13)$ & $\mathrm{C} 16-\mathrm{C} 15-\mathrm{H} 15$ & 120.4 \\
\hline $\mathrm{C} 5-\mathrm{C} 6-\mathrm{H} 6$ & 119.7 & $\mathrm{C} 14-\mathrm{C} 15-\mathrm{H} 15$ & 120.4 \\
\hline $\mathrm{C} 12-\mathrm{C} 6-\mathrm{H} 6$ & 119.7 & $\mathrm{C} 15-\mathrm{C} 16-\mathrm{C} 17$ & $121.63(13)$ \\
\hline $\mathrm{C} 8-\mathrm{C} 7-\mathrm{C} 12$ & $120.26(13)$ & $\mathrm{C} 15-\mathrm{C} 16-\mathrm{Cl1}$ & $119.34(12)$ \\
\hline $\mathrm{C} 8-\mathrm{C} 7-\mathrm{H} 7$ & 119.9 & $\mathrm{C} 17-\mathrm{C} 16-\mathrm{Cl} 1$ & $119.02(12)$ \\
\hline $\mathrm{C} 12-\mathrm{C} 7-\mathrm{H} 7$ & 119.9 & $\mathrm{C} 16-\mathrm{C} 17-\mathrm{C} 18$ & $118.81(14)$ \\
\hline $\mathrm{C} 7-\mathrm{C} 8-\mathrm{C} 9$ & $122.29(13)$ & $\mathrm{C} 16-\mathrm{C} 17-\mathrm{H} 17$ & 120.6 \\
\hline $\mathrm{C} 7-\mathrm{C} 8-\mathrm{H} 8$ & 118.9 & $\mathrm{C} 18-\mathrm{C} 17-\mathrm{H} 17$ & 120.6 \\
\hline $\mathrm{C} 9-\mathrm{C} 8-\mathrm{H} 8$ & 118.9 & $\mathrm{C} 17-\mathrm{C} 18-\mathrm{C} 13$ & $120.32(14)$ \\
\hline $\mathrm{C} 10-\mathrm{C} 9-\mathrm{C} 8$ & $118.85(13)$ & $\mathrm{C} 17-\mathrm{C} 18-\mathrm{H} 18$ & 119.8 \\
\hline $\mathrm{C} 10-\mathrm{C} 9-\mathrm{H} 9$ & 120.6 & $\mathrm{C} 13-\mathrm{C} 18-\mathrm{H} 18$ & 119.8 \\
\hline $\mathrm{C} 8-\mathrm{C} 9-\mathrm{H} 9$ & 120.6 & & \\
\hline $\mathrm{C} 13-\mathrm{N} 1-\mathrm{C} 1-\mathrm{C} 10$ & $4.4(2)$ & $\mathrm{C} 2-\mathrm{C} 3-\mathrm{C} 11-\mathrm{C} 10$ & $0.57(17)$ \\
\hline $\mathrm{C} 13-\mathrm{N} 1-\mathrm{C} 1-\mathrm{C} 2$ & $-177.46(12)$ & $\mathrm{C} 9-\mathrm{C} 10-\mathrm{C} 11-\mathrm{C} 12$ & $-0.1(2)$ \\
\hline $\mathrm{N} 1-\mathrm{C} 1-\mathrm{C} 2-\mathrm{O} 1$ & $3.7(2)$ & $\mathrm{C} 1-\mathrm{C} 10-\mathrm{C} 11-\mathrm{C} 12$ & $-178.59(13)$ \\
\hline
\end{tabular}




$$
\begin{aligned}
& \mathrm{C} 10-\mathrm{C} 1-\mathrm{C} 2-\mathrm{O} 1 \\
& \mathrm{~N} 1-\mathrm{C} 1-\mathrm{C} 2-\mathrm{C} 3 \\
& \mathrm{C} 10-\mathrm{C} 1-\mathrm{C} 2-\mathrm{C} 3 \\
& \mathrm{O} 1-\mathrm{C} 2-\mathrm{C} 3-\mathrm{C} 4 \\
& \mathrm{C} 1-\mathrm{C} 2-\mathrm{C} 3-\mathrm{C} 4 \\
& \mathrm{O} 1-\mathrm{C} 2-\mathrm{C} 3-\mathrm{C} 11 \\
& \mathrm{C} 1-\mathrm{C} 2-\mathrm{C} 3-\mathrm{C} 11 \\
& \mathrm{C} 11-\mathrm{C} 3-\mathrm{C} 4-\mathrm{C} 5 \\
& \mathrm{C} 2-\mathrm{C} 3-\mathrm{C} 4-\mathrm{C} 5 \\
& \mathrm{C} 3-\mathrm{C} 4-\mathrm{C} 5-\mathrm{C} 6 \\
& \mathrm{C} 4-\mathrm{C} 5-\mathrm{C} 6-\mathrm{C} 12 \\
& \mathrm{C} 12-\mathrm{C} 7-\mathrm{C} 8-\mathrm{C} 9 \\
& \mathrm{C} 7-\mathrm{C} 8-\mathrm{C} 9-\mathrm{C} 10 \\
& \mathrm{C} 8-\mathrm{C} 9-\mathrm{C} 10-\mathrm{C} 11 \\
& \mathrm{C} 8-\mathrm{C} 9-\mathrm{C} 10-\mathrm{C} 1 \\
& \mathrm{~N} 1-\mathrm{C} 1-\mathrm{C} 10-\mathrm{C} 9 \\
& \mathrm{C} 2-\mathrm{C} 1-\mathrm{C} 10-\mathrm{C} 9 \\
& \mathrm{~N} 1-\mathrm{C} 1-\mathrm{C} 10-\mathrm{C} 11 \\
& \mathrm{C} 2-\mathrm{C} 1-\mathrm{C} 10-\mathrm{C} 11 \\
& \mathrm{C} 4-\mathrm{C} 3-\mathrm{C} 11-\mathrm{C} 12
\end{aligned}
$$

$-177.65(14)$

$-177.25(13)$

$1.36(14)$

$-3.2(3)$

$177.87(15)$

$177.79(15)$

$-1.18(15)$

-0.4 (2)

$-179.39(14)$

$0.0(2)$

$0.6(2)$

$-0.3(3)$

$0.0(2)$

$0.2(2)$

$178.14(16)$

-0.8 (3)

$-179.12(16)$

$177.30(16)$

$-1.04(15)$

$0.3(2)$

$179.49(13)$

$-178.61(13)$

$$
\begin{aligned}
& \mathrm{C} 9-\mathrm{C} 10-\mathrm{C} 11-\mathrm{C} 3 \\
& \mathrm{C} 1-\mathrm{C} 10-\mathrm{C} 11-\mathrm{C} 3 \\
& \mathrm{C} 3-\mathrm{C} 11-\mathrm{C} 12-\mathrm{C} 7 \\
& \mathrm{C} 10-\mathrm{C} 11-\mathrm{C} 12-\mathrm{C} 7 \\
& \mathrm{C} 3-\mathrm{C} 11-\mathrm{C} 12-\mathrm{C} 6 \\
& \mathrm{C} 10-\mathrm{C} 11-\mathrm{C} 12-\mathrm{C} 6 \\
& \mathrm{C} 8-\mathrm{C} 7-\mathrm{C} 12-\mathrm{C} 11 \\
& \mathrm{C} 8-\mathrm{C} 7-\mathrm{C} 12-\mathrm{C} 6 \\
& \mathrm{C} 5-\mathrm{C} 6-\mathrm{C} 12-\mathrm{C} 11 \\
& \mathrm{C} 5-\mathrm{C} 6-\mathrm{C} 12-\mathrm{C} 7 \\
& \mathrm{C} 1-\mathrm{N} 1-\mathrm{C} 13-\mathrm{C} 18 \\
& \mathrm{C} 1-\mathrm{N} 1-\mathrm{C} 13-\mathrm{C} 14 \\
& \mathrm{C} 18-\mathrm{C} 13-\mathrm{C} 14-\mathrm{C} 15 \\
& \mathrm{~N} 1-\mathrm{C} 13-\mathrm{C} 14-\mathrm{C} 15 \\
& \mathrm{C} 13-\mathrm{C} 14-\mathrm{C} 15-\mathrm{C} 16 \\
& \mathrm{C} 14-\mathrm{C} 15-\mathrm{C} 16-\mathrm{C} 17 \\
& \mathrm{C} 14-\mathrm{C} 15-\mathrm{C} 16-\mathrm{C} 11 \\
& \mathrm{C} 15-\mathrm{C} 16-\mathrm{C} 17-\mathrm{C} 18 \\
& \mathrm{C} 11-\mathrm{C} 16-\mathrm{C} 17-\mathrm{C} 18 \\
& \mathrm{C} 16-\mathrm{C} 17-\mathrm{C} 18-\mathrm{C} 13 \\
& \mathrm{C} 14-\mathrm{C} 13-\mathrm{C} 18-\mathrm{C} 17 \\
& \mathrm{~N} 1-\mathrm{C} 13-\mathrm{C} 18-\mathrm{C} 17
\end{aligned}
$$

$$
\begin{aligned}
& 178.77(13) \\
& 0.33(17) \\
& -178.99(13) \\
& -0.2(2) \\
& 0.3(2) \\
& 179.11(13) \\
& 0.4(2) \\
& -178.80(16) \\
& -0.7(2) \\
& 178.45(15) \\
& 81.00(18) \\
& -102.89(16) \\
& -3.6(2) \\
& -179.75(12) \\
& 1.3(2) \\
& 1.9(2) \\
& -179.20(10) \\
& -2.7(2) \\
& 178.37(10) \\
& 0.3(2) \\
& 2.8(2) \\
& 178.94(12)
\end{aligned}
$$

Hydrogen-bond geometry $\left(\AA,{ }^{\circ}\right)$

$\mathrm{Cg}$ is the centroid of the 4-chlorophenyl ring (C13-C18).

\begin{tabular}{lllll}
\hline$D-\mathrm{H} \cdots A$ & $D-\mathrm{H}$ & $\mathrm{H} \cdots A$ & $D \cdots A$ & $D-\mathrm{H} \cdots A$ \\
\hline $\mathrm{C} 7-\mathrm{H} 7 \cdots \mathrm{Cl1}{ }^{\mathrm{i}}$ & 0.95 & 2.80 & $3.748(2)$ & 179 \\
$\mathrm{C} 6-\mathrm{H} 6 \cdots C g^{\mathrm{ii}}$ & 0.95 & 2.75 & $3.698(4)$ & 177 \\
$\mathrm{C} 9-\mathrm{H} 9 \cdots C g$ & 0.95 & 2.87 & $3.644(4)$ & 142 \\
\hline
\end{tabular}

Symmetry codes: (i) $x, y, z+1$; (ii) $x+1, y, z+1$. 